\title{
Feasibility of biohydrogen production from industrial wastes using defined microbial co-culture
}

Peng Chen ${ }^{1 * \dagger}$, Yuxia Wang ${ }^{3 \dagger}$, Lei Yan ${ }^{4}$, Yiqing Wang ${ }^{5}$, Suyue Li ${ }^{2}$, Xiaojuan Yan ${ }^{2}$, Ningbo Wang ${ }^{2}$, Ning Liang ${ }^{2}$ and Hongyu Li ${ }^{* *}$

\begin{abstract}
Background: The development of clean or novel alternative energy has become a global trend that will shape the future of energy. In the present study, 3 microbial strains with different oxygen requirements, including Clostridium acetobutylicum ATCC 824, Enterobacter cloacae ATCC 13047 and Kluyveromyces marxianus 15D, were used to construct a hydrogen production system that was composed of a mixed aerobic-facultative anaerobic-anaerobic consortium. The effects of metal ions, organic acids and carbohydrate substrates on this system were analyzed and compared using electrochemical and kinetic assays. It was then tested using small-scale experiments to evaluate its ability to convert starch in $5 \mathrm{~L}$ of organic wastewater into hydrogen. For the one-step biohydrogen production experiment, $\mathrm{H} 1$ medium (nutrient broth and potato dextrose broth) was mixed directly with GAM broth to generate $\mathrm{H} 2$ medium (H1 medium and GAM broth). Finally, Clostridium acetobutylicum ATCC 824, Enterobacter cloacae ATCC 13047 and Kluyveromyces marxianus 15D of three species microbial co-culture to produce hydrogen under anaerobic conditions. For the two-step biohydrogen production experiment, the $\mathrm{H} 1$ medium, after cultured the microbial strains Enterobacter cloacae ATCC 13047 and Kluyveromyces marxianus 15D, was centrifuged to remove the microbial cells and then mixed with GAM broth (H2 medium). Afterward, the bacterial strain Clostridium acetobutylicum ATCC 824 was inoculated into the $\mathrm{H} 2$ medium to produce hydrogen by anaerobic fermentation.

Results: The experimental results demonstrated that the optimum conditions for the small-scale fermentative hydrogen production system were at $\mathrm{pH} 7.0,35^{\circ} \mathrm{C}$, a mixed medium, including $\mathrm{H} 1$ medium and $\mathrm{H} 2$ medium with $0.50 \mathrm{~mol} / \mathrm{L}$ ferrous chloride, $0.50 \mathrm{~mol} / \mathrm{L}$ magnesium sulfate, $0.50 \mathrm{~mol} / \mathrm{L}$ potassium chloride, $1 \% \mathrm{w} / \mathrm{V}$ citric acid, $5 \% \mathrm{w} / \mathrm{V}$ fructose and $5 \% \mathrm{w} / \mathrm{v}$ glucose. The overall hydrogen production efficiency in the shake flask fermentation group was $33.7 \mathrm{~mL} / \mathrm{h}^{-1} \cdot \mathrm{L}^{-1}$, and those the two-step and the one-step processes of the small-scale fermentative hydrogen production system were $41.2 \mathrm{~mL} / \mathrm{h}^{-1} \cdot \mathrm{L}^{-1}$ and $35.1 \mathrm{~mL} / \mathrm{h}^{-1} \cdot \mathrm{L}^{-1}$, respectively.
\end{abstract}

Conclusion: Therefore, the results indicate that the hydrogen production efficiency of the two-step process is higher than that of the one-step process.

Keywords: Renewable Energy, Biohydrogen, Microbial consortium, Hydrogen

\footnotetext{
* Correspondence: chenpeng@|zu.edu.cn; lihy@|zu.edu.cn

${ }^{\dagger}$ Equal contributors

'School of Pharmacy, Lanzhou University, Donggang West Road No. 199, Lanzhou 730020, People's Republic of China

Full list of author information is available at the end of the article
} 


\section{Background}

The research and development of hydrogen energy has attracted worldwide attention and become an important strategy for the production of clean energy [1,2]. Among the various technologies that have been developed for hydrogen production, the utilization of hydrogenproducing microbes is one of the most effective. Currently, microbial fermentative hydrogen production is achieved mainly through 2 routes: one utilizes pure microbial strains and the other employs a mixed microbial consortium [3-6]. Researchers have performed a large number of studies on fermentative hydrogen production using pure microbial strains and its disadvantages, including short test cycles, low hydrogen yields and poor applicability in the presence of complex substrates. To take better advantage of low-cost organic substrates for hydrogen production, recycle wastes and improve the environment, in recent years, many researchers have focused on hydrogen production by microbial fermentation using a mixed microbial consortium, in which various microbial strains exert synergistic effects $[7,8]$. In a favorable living environment that fully supports metabolic activities, the hydrogen production capacity and hydrogen yield from the mixed microbial consortium were enhanced compared with those of the pure microbial strains. Recent studies have confirmed that the hydrogen production efficiency of mixed microbial consortia are significantly higher than that of individual pure strains $[9,10]$. Therefore, the production of hydrogen by fermentation using low-cost agricultural waste by a mixed microbial consortium is a novel development for the hydrogen energy industry [11].

Our previous work was to observe the hydrogen production yield of 16 different pure strains and mixed culture, which contains hydrogen-producing strains and nonhydrogen-producing strains. The experimental results compared the hydrogen production yield of these pure strains. Based on these results and on the comparative hydrogen-production efficiencies of the microbial strains, Enterobacter cloacae (E. cloacae) ATCC 13047, Kluyveromyces marxianus (K. marxianus) 15D and Clostridium acetobutylicum (C. acetobutylicum) ATCC 824 were identified as suitable for use in hydrogen-producing mixed microfloras. In the present study, by the application of combinatorially optimized culture media, 3 microbial strains with different oxygen requirements, including C. acetobutylicum ATCC 824, E. cloacae ATCC 13047 and $K$. marxianus $15 \mathrm{D}$, were combined to construct a hydrogen production system that was composed of a mixed aerobic-facultative anaerobic-anaerobic consortium.

The metal ions, organic acids and carbohydrate substrates are the most common material in industrial wastes environment. The ability of microorganism to produce hydrogen in the presence of industrial wastes sources (metal ions, organic acids and carbohydrate substrates) is worthy of study. The fundamental knowledge derived from this study should provide a valuable platform for further investigation into the behavior of microorganism involved in hydrogen production system and has potential biotechnological applications in waste resources reused. Therefore, the effects of metal ions, organic acids and carbohydrate substrates on the system were analyzed by assaying the electrochemical and kinetic parameters, along with the optimization and quality control of hydrogen production process. Based on the identification of the factors affecting the hydrogen production process, a small-scale biohydrogen production system (5 L) using starchy organic wastewater was constructed with the intent of providing preliminary data for further pilot-scale biohydrogen production.

\section{Results and discussion}

\section{Effects of metal ions on hydrogen production system composed of mixed microbial consortium}

To determine the effects of various metal ions on the $\mathrm{H} 1$ medium- and $\mathrm{H} 2$ medium-based hydrogen production systems, a two-step system that was composed of the 3 microbial strains was studied. The experimental results are shown in Table 1. In this experiment, the controls correspond to cultures with no supplemented metal ions. Table 1 demonstrates that $\mathrm{Fe}^{2+}$ had stimulatory effects on the hydrogen production system that was composed of the mixed microbial strains E. cloacae ATCC 13047 and K. marxianus 15D, while $\mathrm{Fe}^{3+}$ activated the system that was composed of the bacterial strain C. acetobutylicum ATCC 824. Previous studies have shown that at the cellular level, certain metal ions affect the activity and quantity of hydrogen-producing bacteria. It has been shown that iron deficiency not only affects the growth and metabolism of the ethanol-based hydrogen-producing fermentative bacterium B49 but also its ability to produce hydrogen. The addition of $\mathrm{Fe}^{2+}$ to a culture of hydrogen-producing fermentative bacteria has been shown to stimulate the specific activities of hydrogenase and NADH-Fe reductase, enhancing its hydrogen production activities and improving its hydrogen production capacities [12].

Static fermentation experiments using 20 hydrogenproducing bacterial strains have shown that the addition of ferrum to culture media causes a shift from butyric acid to ethanol fermentation. In the 2 main pathways of fermentative hydrogen production from organic materials, ferrum is one of the essential components that participate in and promote various enzymatic reactions. Under similar culture conditions, both $\mathrm{Fe}^{3+}$ and $\mathrm{Fe}^{2+}$ stimulate the conversion of bacterial metabolism to ethanol-type fermentation, and $\mathrm{Fe}^{3+}$ exhibits stronger effects than $\mathrm{Fe}^{2+}$. Furthermore, 
Table 1 The effects of different metal ions on the hydrogen production systems

\begin{tabular}{llll}
\hline Metal ions $(\mathbf{0 . 5 0} \mathbf{~ m o l} / \mathbf{L})$ & $\mathbf{H} \mathbf{~ m e d i u m}\left(\mathbf{m L} / \mathbf{h}^{-\mathbf{1}} \cdot \mathbf{L}^{-\mathbf{1}}\right)$ & $\mathbf{H} \mathbf{~ m e d i u m}\left(\mathbf{m L} / \mathbf{h}^{-1} \cdot \mathbf{L}^{\mathbf{- 1}}\right)$ & Overall hydrogen production $(\%)$ \\
\hline Controls & $17.80 \pm 1.51$ & $15.90 \pm 0.66$ & 100.0 \\
$\mathrm{BaCl}_{2} \cdot 2 \mathrm{H}_{2} \mathrm{O}$ & $3.74 \pm 1.04$ & $2.07 \pm 0.36$ & 17.21 \\
$\mathrm{CaCl}_{2}$ & $6.23 \pm 0.25$ & $4.29 \pm 1.03$ & 31.16 \\
$\mathrm{CoCl}_{2} \cdot 6 \mathrm{H}_{2} \mathrm{O}$ & $2.14 \pm 0.99$ & $1.113 \pm 1.04$ & 9.50 \\
$\mathrm{FeCl}_{2} \cdot 4 \mathrm{H}_{2} \mathrm{O}$ & $21.54 \pm 1.02$ & $13.67 \pm 0.76$ & 104.45 \\
$\mathrm{FeCl}_{3} \cdot 4 \mathrm{H}_{2} \mathrm{O}$ & $14.06 \pm 1.09$ & $18.44 \pm 0.58$ & 96.44 \\
$\mathrm{CuSO}_{4} \cdot 5 \mathrm{H}_{2} \mathrm{O}$ & $14.77 \pm 1.17$ & $10.65 \pm 1.97$ & 75.37 \\
$\mathrm{KCl}$ & $18.69 \pm 0.23$ & $15.90 \pm 1.54$ & 102.67 \\
$\mathrm{MnSO}_{4} \cdot \mathrm{H}_{2} \mathrm{O}$ & $13.53 \pm 0.76$ & $10.02 \pm 0.15$ & 69.73 \\
$\mathrm{MgSO}_{4} \cdot 7 \mathrm{H}_{2} \mathrm{O}$ & $21.18 \pm 0.16$ & $20.19 \pm 0.83$ & 122.85 \\
$\mathrm{NH}_{4} \mathrm{Cl}$ & $12.64 \pm 1.88$ & $10.81 \pm 1.96$ & 69.73 \\
$\mathrm{ZnSO}_{4} \cdot 7 \mathrm{H}_{2} \mathrm{O}$ & $16.20 \pm 1.09$ & $12.08 \pm 1.03$ & 83.98 \\
\hline
\end{tabular}

$\mathrm{Fe}^{2+}$ enhances the fermentative hydrogen production capacities of bacteria [13].

In addition, $\mathrm{Mg}^{2+}$ and $\mathrm{K}^{+}$ions also exhibit stimulatory effects on hydrogen production systems. $\mathrm{Mg}^{2+}$ is an important influential factor. Of the 10 types of cytoplasmic enzymes that are required for glycolysis, a vast majority are activated by $\mathrm{Mg}^{2+}$, and its deficiency affects the growth and anabolism of hydrogen-producing bacteria, thereby affecting the ability of the bacteria to produce hydrogen. The addition of $\mathrm{Mg}^{2+}$ to culture media has been shown to promote the growth of ethanol-based hydrogen-producing fermentative bacteria and thus enhance their hydrogen production capacities [14]. In contrast, other metal ions, such as $\mathrm{Ba}^{2+}, \mathrm{Ca}^{2+}, \mathrm{Co}^{2+}, \mathrm{Cu}^{2+}$, $\mathrm{Mn}^{2+}$ and $\mathrm{Zn}^{2+}$, appear to exert inhibitory effects on hydrogen production systems by hindering microbial growth and hydrogenase activities.

Effects of organic acids on hydrogen production systems composed of mixed microbial consortium

To determine the effects of various organic acids on H1 medium- and H2 medium-based hydrogen production systems, a two-step system that was composed of the 3 microbial strains was studied. Table 2 shows that citric acid significantly enhances hydrogen production efficiencies, whereas the remaining organic acids that were tested displayed inhibitory effects.
Effects of carbohydrate substrates on hydrogen production systems composed of mixed microbial consortium

To determine the effects of various carbohydrate substrates on $\mathrm{H} 1$ medium- and $\mathrm{H} 2$ medium-based hydrogen production systems, a two-step system that was composed of the 3 microbial strains was studied. As shown in Table 2, both fructose and glucose all exert significant stimulatory effects on the system, whereas the other carbohydrate substrates used in this study exhibited strong inhibition.

\section{Small-scale biohydrogen production experiments based} on starchy organic wastewater

Construction of mixed microbial consortium for biohydrogen production and synergistic effects between microbial strains

The biohydrogen-producing mixed microbial consortium was constructed using 3 hydrogen-producing microbial strains, including C. acetobutylicum ATCC 824, E. cloacae ATCC 13047 and K. marxianus 15D, and its electrochemical characteristics were analyzed. The hydrogen production efficiency of the mixed aerobic-facultative anaerobic consortium was found to be significantly higher than those of the hydrogen production systems that were composed of individual pure microbial strains (Figure 1). The hydrogen production efficiencies of E. cloacae ATCC 13047 and K. marxianus 15D were $12.5 \mathrm{~mL} / \mathrm{h}^{-1} \cdot \mathrm{L}^{-1}$ and $8.9 \mathrm{~mL} / \mathrm{h}^{-1} \cdot \mathrm{L}^{-1}$,

Table 2 The effects of various organic acids on the hydrogen production efficiencies

\begin{tabular}{|c|c|c|c|}
\hline Organic acids $(1 \% \mathrm{w} / \mathrm{v})$ & $\mathrm{H} 1$ medium $\left(\mathrm{mL} / \mathrm{h}^{-1} \cdot \mathrm{L}^{-1}\right)$ & $\mathrm{H} 2$ medium $\left(\mathrm{mL} / \mathrm{h}^{-1} \cdot \mathrm{L}^{-1}\right)$ & Overall hydrogen production (\%) \\
\hline Controls & $17.80 \pm 1.51$ & $15.90 \pm 0.66$ & 100.0 \\
\hline Acetic acid & $6.20 \pm 2.70$ & $7.60 \pm 1.60$ & 40.95 \\
\hline Citric acid & $18.0 \pm 1.10$ & $20.3 \pm 1.02$ & 113.65 \\
\hline Ethacetic acid & $8.10 \pm 3.12$ & $8.0 \pm 2.15$ & 47.77 \\
\hline Lactic acid & $19.0 \pm 2.03$ & $10.1 \pm 1.02$ & 86.35 \\
\hline Oxalic acid & $12.0 \pm 3.98$ & $12 \pm 1.25$ & 71.22 \\
\hline
\end{tabular}




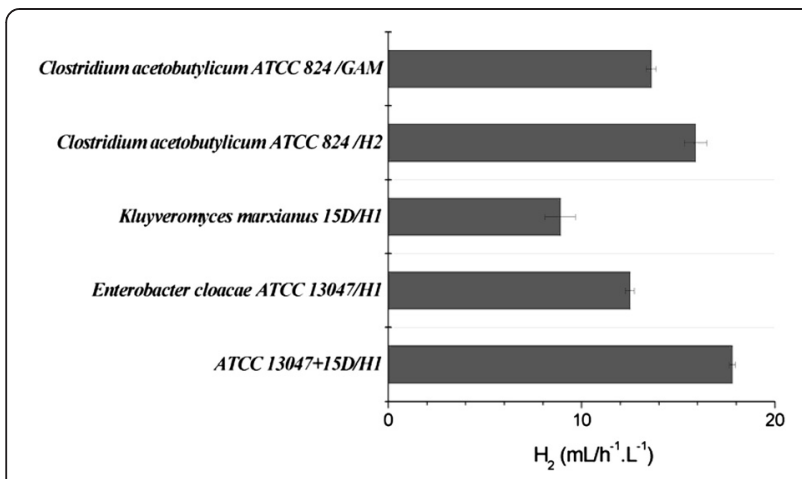

Figure 1 Analysis and comparison of the hydrogen production efficiencies of the mixed microbial consortia.

respectively, whereas that of the mixed microbial consortium that was composed of the 2 microbial strains reached $17.8 \mathrm{~mL} / \mathrm{h}^{-1} \cdot \mathrm{L}^{-1}$. Therefore, the efficiency of the mixed microbial consortium was significantly enhanced compared to those of the pure microbial strains. The key determinant of whether a culture medium ( $\mathrm{H} 2$ medium) for the aerobicfacultative anaerobic-anaerobic consortium could be successfully developed was whether the H1 medium that was harvested from the cultured microbial stains E. cloacae ATCC 13047 and K. marxianus 15D was able to support the growth of the bacterial strain C. acetobutylicum ATCC 824. Thus, C. acetobutylicum ATCC 824 was inoculated into the $\mathrm{H} 2$ liquid medium, and the electrochemical parameters of the culture were determined. The hydrogen production efficiency of $C$. acetobutylicum ATCC 824 in $\mathrm{H} 2$ medium was found to be significantly higher than that of the hydrogen production system that was composed of the pure culture of $C$. acetobutylicum ATCC 824. As shown in Figure 2, the efficiency of C. acetobutylicum ATCC 824 was $13.6 \mathrm{~mL} / \mathrm{h}^{-1} \cdot \mathrm{L}^{-1}$ in GAM broth, whereas it was $15.9 \mathrm{~mL} / \mathrm{h}^{-1} \cdot \mathrm{L}^{-1}$ in $\mathrm{H} 2$ medium. The increased efficiency

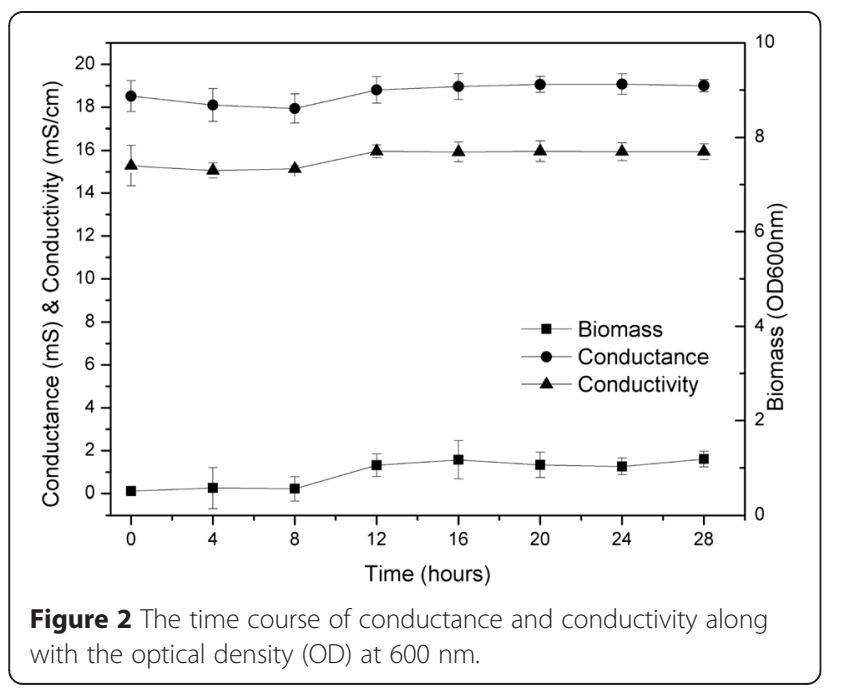

that was observed in the $\mathrm{H} 2$ medium was probably due to the efficient degradation of starch in the $\mathrm{H} 1$ medium by the microbial strains E. cloacae ATCC 13047 and K. marxianus 15D. The $\mathrm{H} 2$ medium was constructed using the $\mathrm{H} 1$ medium as a base. Therefore, $C$. acetobutylicum ATCC 824 was able to better utilize the substrates, effectively converting glucose and other substances to hydrogen via the catalytic activity of hydrogenase. Thus, the efficiency of the two-step hydrogen production system that was composed of the mixed microbial consortium was significantly higher than that of the systems that were composed of the pure anaerobic strains $[15,16]$.

\section{Effects of 2 types of biohydrogen production processes on electrochemical properties of hydrogen production systems}

The electrochemical parameters of the two-step and one-step hydrogen production systems were compared (Figure 2). In hydrogen-production systems composed of pure microbial strains, when all other conditions are constant, the electrical conductance of the biohydrogenproducing solution is determined by the number of ions, their electric charges, and their mobility. The electrical conductance of the biohydrogen-producing solution is the sum of the electrical conductances of the various ions in the solution. Therefore, in the biological hydrogenproduction process, the electrical conductance and conductivity can be utilised to determine the concentrations of the components in a hydrogen-production system and to optimise hydrogen-production conditions.

The results showed the following: (1) in the one-step hydrogen production system, a gradual declining trend in $\mathrm{pH}$ was observed, whereas in the two-step system, an opposite trend was observed; (2) in the one-step hydrogen production system, the redox potential showed a gradual rising trend, increasing from $25 \mathrm{mV}$ to $89 \mathrm{mV}$. In contrast, the redox potential of the two-step system decreased gradually within a small range; (3) the electrical conductance and conductivity of the one-step hydrogen production system were higher than those of the two-step system.

\section{Effects of 2 types of biohydrogen production processes on hydrogen production efficiency}

The efficiencies of the 2 types of biohydrogen production processes were compared and analyzed. The results are shown in Figure 3. The shake flask fermentation group, which is abbreviated as $\mathrm{Y}$ in Figure 3, was used as the control. The two-step hydrogen production process is abbreviated as $2 \mathrm{~F}$, and the one-step process is abbreviated as $1 \mathrm{~F}$.

As shown in Figure 3, the overall hydrogen production efficiency in the shake flask fermentation group was $33.7 \mathrm{~mL} / \mathrm{h}^{-1} \cdot \mathrm{L}^{-1}$, and those the two-step and the one-step processes were $41.2 \mathrm{~mL} / \mathrm{h}^{-1} \cdot \mathrm{L}^{-1}$ and $35.1 \mathrm{~mL} / \mathrm{h}^{-1} \cdot \mathrm{L}^{-1}$, 


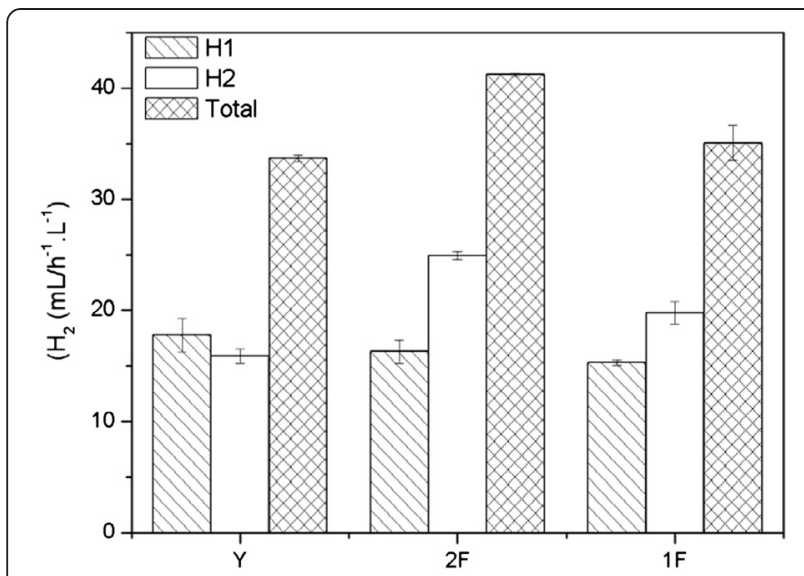

Figure $\mathbf{3}$ The effects of the 2 types of biohydrogen production processes on the hydrogen production efficiency.

respectively, which were higher than that of the shake flask fermentation group. Therefore, the performance of the small-scale fermentative hydrogen production system was superior to the shake flask fermentation method. In addition, the overall hydrogen production efficiency of the two-step process was higher than that of the one-step process. The differences in the overall hydrogen production efficiencies were related to the removal of the centrifugation step.

\section{Microscopic analysis of mixed microbial consortium}

The microscopic images of the mixed microbial consortium are shown in Figure 4. It was revealed that the microbial strains E. cloacae ATCC 13047 and K. marxianus 15D could be co-cultured in $\mathrm{H} 1$ liquid medium. K. marxianus 15D utilized starch for biomass production more efficiently than E. cloacae ATCC 13047. Therefore, compared to E. cloacae ATCC 13047 (Microbial shape is small rod, pointed out by the black arrow in Figure 4A), significantly larger numbers of $K$. marxianus 15D (Yeast shape is big sphere, denoted by the black circle in Figure 4A) were observed microscopically. Additionally, Figure $4 \mathrm{~B}$ showed that the bacterial strain C. acetobutylicum ATCC 824 was capable of growing in $\mathrm{H} 2$ medium, and the amount of biomass that was produced by $C$. acetobutylicum ATCC 824 in the $\mathrm{H} 2$ medium (final $\mathrm{OD}_{600 \mathrm{~nm}}=2.3$ ) was elevated compared to that in the GAM broth (final $\mathrm{OD}_{600 \mathrm{~nm}}=2.0$ ). The results indicate that $C$. acetobutylicum ATCC 824 utilized the conditioned $\mathrm{H} 1$ liquid medium that was harvested from the culture of E. cloacae ATCC 13047 and $K$. marxianus 15D more efficiently, significantly improving the hydrogen production efficiency.

\section{Conclusion}

The mixed microbial consortium was constructed by a two-step process using 3 microbial strains, including $C$. acetobutylicum ATCC 824, E. cloacae ATCC 13047 and $K$. marxianus $15 \mathrm{D}$, and exhibited a high hydrogen production efficiency. Analyses of the effects of various metal ions, organic acids and carbohydrate substrates on the hydrogen production systems showed that the $\mathrm{Fe}^{2+} / \mathrm{Fe}^{3+}$ ions were crucial for the systems. $\mathrm{Mg}^{2+}$ and $\mathrm{K}^{+}$also exerted stimulatory effects. Citric acid significantly enhanced hydrogen production efficiency. Additionally, fructose and glucose exhibited significant stimulatory effects. The hydrogen production efficiency of the two-step process is higher than that of the one-step process.

\section{Methods}

\section{Microorganism}

The microbial strains, used in the present study, were $C$. acetobutylicum ATCC 824, E. cloacae ATCC 13047 and K. marxianus $15 \mathrm{D}$. All the three strains were preserved in our laboratory.

\section{Medium composition}

\section{The composition of the nutrient broth was as follows}

$10.0 \mathrm{~g}$ of peptone, $3.0 \mathrm{~g}$ of beef extract, $5.0 \mathrm{~g}$ of $\mathrm{NaCl}$, $20.0 \mathrm{~g}$ of agar and 1.0 $\mathrm{L}$ of distilled water. The medium was adjusted to $\mathrm{pH} 7.0$ with $5 \mathrm{~mol} / \mathrm{L}$ sodium hydroxide (approximately $0.2 \mathrm{~mL}$ ) and autoclaved at $1.05 \mathrm{Kg} / \mathrm{cm}^{2}$ for $20 \mathrm{~min}$.

The composition of the potato dextrose broth was as follows: $200.0 \mathrm{~g}$ of potato, $20.0 \mathrm{~g}$ of dextrose, $1.0 \mathrm{~L}$ of distilled water, $\mathrm{pH}$ 7.0. The potatoes were washed, peeled

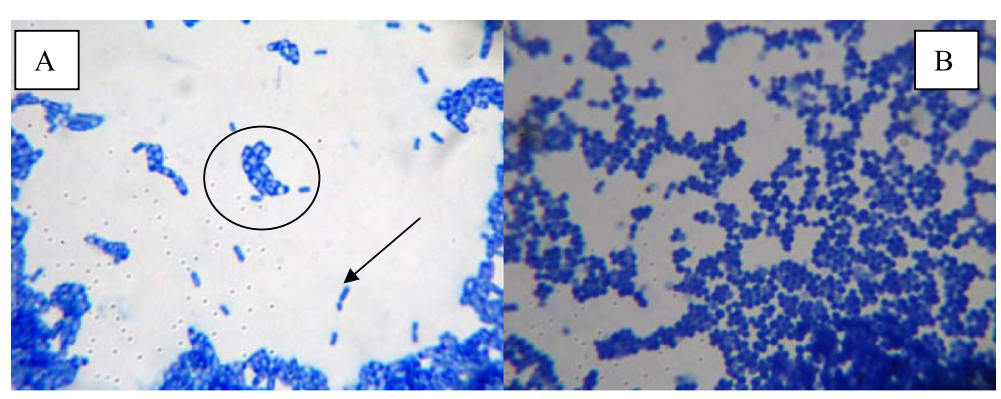

Figure 4 Microscopic morphologies of the microbial cells in mixed culture media (A) C. acetobutylicum ATCC 824 and E. cloacae ATCC 13047 in H1 medium; (B) C. acetobutylicum ATCC 824 in H2 medium. 
and sliced. The potato dextrose agar (PDA) medium was prepared from $200 \mathrm{~g}$ washed and sliced potatoes, boiled in $500 \mathrm{ml}$ filtered and strained through gauze. $20.0 \mathrm{~g}$ Agar was melted in the solution, and $0.5 \mathrm{~L}$ water and $20.0 \mathrm{~g}$ glucose were added before the medium was aliquoted and autoclaved at $1.05 \mathrm{Kg} / \mathrm{cm}^{2}$ for $20 \mathrm{~min}$ [17].

\section{The aerobic-facultative anaerobic culture medium (abbreviation: $\mathrm{H} 1$ medium) was prepared as follows}

The nutrient broth and potato dextrose agar were prepared separately as described above and mixed evenly. The medium was utilized for the routine culture of the microbial strains E. cloacae ATCC 13047 and K. marxianus 15D.

The composition of the modified Gifu anaerobic medium (GAM) broth was as follows: $15.0 \mathrm{~g}$ of proteose peptone, $10.0 \mathrm{~g}$ of pancreatic casein peptone, $5.0 \mathrm{~g}$ of yeast extract, $2.0 \mathrm{~g}$ of beef powder, $13.5 \mathrm{~g}$ of digestive serum powder, $1.2 \mathrm{~g}$ of bovine liver extract powder, $3.0 \mathrm{~g}$ of glucose, $2.5 \mathrm{~g}$ of potassium dihydrogen phosphate, $3.0 \mathrm{~g}$ of sodium chloride, $0.3 \mathrm{~g}$ of soluble starch, $0.3 \mathrm{~g}$ of L-cysteine and $0.15 \mathrm{~g}$ of sodium thioglycolate. The medium was prepared by adding $1.0 \mathrm{~L}$ of distilled water to $74.0 \mathrm{~g}$ of modified GAM broth and autoclaved at $1.05 \mathrm{Kg} / \mathrm{cm}^{2}$ for $20 \mathrm{~min}$.

\section{The mixed culture medium (abbreviated: $\mathrm{H} 2$ medium) was prepared as follows}

The microbial strains E. cloacae ATCC 13047 and K. marxianus 15D were inoculated into the $\mathrm{H} 1$ medium. After an incubation time of $30 \mathrm{~h}$, the microbial cultures were centrifuged at $10,000 \mathrm{r} / \mathrm{min}$ for $10 \mathrm{~min}$, and the liquid medium were collected. The $\mathrm{H} 2$ medium was obtained by adding $74.0 \mathrm{~g}$ of modified GAM broth to $1.0 \mathrm{~L}$ of liquid medium, which was then autoclaved at $1.05 \mathrm{Kg} /$ $\mathrm{cm}^{2}$ for $20 \mathrm{~min}$. The $\mathrm{H} 2$ medium was used to culture the bacterial strain C. acetobutylicum ATCC 824

\section{Culture medium for the small-scale biohydrogen production system was prepared as follows}

To produce biohydrogen, the microbial strains were cultured in $\mathrm{H} 1$ medium or $\mathrm{H} 2$ medium. The $\mathrm{H} 1$ medium contained additional components that were not present in the $\mathrm{H} 1 \mathrm{medium}$, including $0.50 \mathrm{~mol} / \mathrm{L}$ ferrous chloride, $0.50 \mathrm{~mol} / \mathrm{L}$ magnesium sulfate, $0.50 \mathrm{~mol} / \mathrm{L}$ potassium chloride, $1 \% \mathrm{w} / \mathrm{v}$ citric acid, $5 \% \mathrm{w} / \mathrm{v}$ fructose and $5 \% \mathrm{w} / \mathrm{v}$ glucose.

\section{Reagents}

The reagents that were used in the present study included peptone (OXIDE), beef extract (BBI), agar (BBI), GAM broth (Qingdao Hi-tech Industrial Park Haibo Biotechnology Co., Ltd.), potatoes, corn flour (commercially available), starch (made in our laboratory from fresh potatoes), lactose, maltose, fructose, glucose and sucrose (all of the above carbohydrates were biochemical reagent grade). All other reagents were analytical grade. An anaerobic gas mixture that was composed of $5 \%(\mathrm{v} / \mathrm{v}) \mathrm{CO}_{2}, 10 \%(\mathrm{v} / \mathrm{v}) \mathrm{H}_{2}$ and $85 \%(\mathrm{v} / \mathrm{v})$ $\mathrm{N}_{2}$ was used as carrier gas. The $\mathrm{N}_{2}$ had a purity level of 99.999\% (v/v). The content of impurities in $\mathrm{N}_{2}$ was as follows: $\mathrm{H}_{2}, \operatorname{ppm}(\mathrm{v} / \mathrm{v}) \leq 1.0, \mathrm{O}_{2}, \mathrm{ppm}(\mathrm{v} / \mathrm{v}) \leq 3.0$ and $\mathrm{H}_{2} \mathrm{O}$, $\operatorname{ppm}(\mathrm{v} / \mathrm{v}) \leq 5.0$.

\section{Experimental procedures}

Effects of various factors on hydrogen production systems

The $\mathrm{H} 1$ medium was prepared by adding various heavy metal ions, organic acids and carbohydrate substrates to the $\mathrm{H} 1$ medium in Tables 1, 2 and 3, respectively. The microbial strains E. cloacae ATCC 13047 and K. marxianus $15 \mathrm{D}$ were inoculated into the $\mathrm{H} 1$ medium, and samples were collected periodically during the incubation period to determine the electrochemical parameters. After an incubation time of $30 \mathrm{~h}$, the microbial cultures were centrifuged, and the supernatants were mixed with modified GAM broth to generate the $\mathrm{H} 2$ medium. The bacterial strain C. acetobutylicum ATCC 824 was inoculated into the $\mathrm{H} 2$ medium and incubated anaerobically. Samples were collected periodically, and the electrochemical parameters were analyzed with the uninoculated $\mathrm{H} 1$ and $\mathrm{H} 2$ media as controls. The results presented were the average of three trials. The results are presented using mean value \pm standard deviation in Tables 1, 2 and 3, respectively.

Table 3 The effects of various carbohydrate substrates on the hydrogen production efficiencies

\begin{tabular}{llll}
\hline Carbohydrate substrates $(\mathbf{5 \%} \mathbf{~ w} / \mathbf{v})$ & $\mathbf{H 1}$ medium $\left(\mathbf{m L} / \mathbf{h}^{-\mathbf{1}} \cdot \mathbf{L}^{-\mathbf{1}}\right)$ & $\mathbf{H} \mathbf{~ m e d i u m}\left(\mathbf{m L} / \mathbf{h}^{-1} \cdot \mathbf{L}^{-\mathbf{1}}\right)$ & Overall hydrogen production $(\%)$ \\
\hline Controls & $17.80 \pm 1.51$ & $15.90 \pm 0.66$ & 100.0 \\
Fructose & $23.3 \pm 1.022$ & $13.3 \pm 1.94$ & 108.61 \\
Glucose & $19 \pm 1.22$ & $16.1 \pm 1.07$ & 104.15 \\
Lactose & $16.4 \pm 2.11$ & $14.3 \pm 1.04$ & 91.10 \\
Maltose & $15.7 \pm 2.01$ & $15.5 \pm 2.64$ & 92.58 \\
Sucrose & $14.9 \pm 2.56$ & $16.7 \pm 1.43$ & 93.77 \\
Starch & $13.8 \pm 2.44$ & $17.3 \pm 2.39$ & 89.32 \\
\hline
\end{tabular}




\section{Small-scale biohydrogen production experiments using starchy organic wastewater}

Construction of a two-step biohydrogen production system In order to obtain an aerobic-facultative anaerobic-anaerobic hydrogen production system in a stepwise manner, the microbial strains E. cloacae ATCC 13047 and $K$. marxianus 15D were inoculated into the H1 medium. Samples were collected periodically during the incubation period to determine the electrochemical parameters. The $\mathrm{H} 2$ medium was constructed from the $\mathrm{H} 1$ medium that was harvested from the cultures of $E$. cloacae ATCC 13047 and K. marxianus 15D. The bacterial strain C. acetobutylicum ATCC 824 was then inoculated into the $\mathrm{H} 2$ medium and incubated anaerobically. Samples were collected periodically during the incubation period, and the electrochemical parameters were analyzed. Uninoculated $\mathrm{H} 1$ and $\mathrm{H} 2$ media served as controls, and the relevant parameters were determined.

For the two-step biohydrogen production experiment, the $\mathrm{H} 1$ medium, after cultured the microbial strains $E$. cloacae ATCC 13047 and K. marxianus 15D, was centrifuged to remove the microbial cells and then mixed with GAM broth (H2 medium). Afterward, the bacterial strain C. acetobutylicum ATCC 824 was inoculated into the $\mathrm{H} 2$ medium to produce hydrogen by anaerobic fermentation.

For the one-step biohydrogen production experiment, the centrifugation step in the two-step hydrogen production process was omitted to ensure the operability of this pilot-scale experiment. The $\mathrm{H} 1$ medium was mixed directly with GAM broth to generate $\mathrm{H} 2$ medium. The bacterial strain $C$. acetobutylicum ATCC 824 was inoculated into the $\mathrm{H} 2$ medium. Finally, three species microbial coculture to produce hydrogen under anaerobic conditions.

Small-scale biohydrogen production experiments The small-scale experiments were conducted in a $5 \mathrm{~L}$ fermentor. The experimental conditions were as follows: liquid volume of $3 \mathrm{~L}$, fermentation temperature of $30^{\circ} \mathrm{C}$, rotation speed of $200 \mathrm{r} / \mathrm{min}$, ventilation rate of $0.5 \mathrm{~L} / \mathrm{min}$, inoculum volume of $10 \%$ and vessel pressure of $0.1 \mathrm{MPa}$. Samples were collected periodically. The yields of hydrogen were determined using the GC-9A gas chromatograph with a thermal conductivity detector (Shimadzu, Japan) with $\mathrm{N}_{2}$ as the carrier gas. The injection volume was $1 \mathrm{~mL}$. Hydrogen concentrations were calculated using the external standard method.

The electrochemical parameters were measured using an FJA-3 electrochemical ion analyzer (Nanjing Zhuan-Di Instrument \& Equipment Co., Ltd.). The fermentation broth was collected periodically, and the temperature, $\mathrm{pH}$, Eh, electrical conductance and conductivity were detected. The measurements were repeated 5 times, and the averages were used for the data analysis. Using the uninoculated liquid medium as a reference, the absorbances of the microbial cultures at the $600 \mathrm{~nm}$ wavelength were measured using a UV-1800PC spectrophotometer (Beijing Purkinje General Instrument Co., Ltd.).

The microbial strains E. cloacae ATCC 13047 and K. marxianus $15 \mathrm{D}$ were inoculated into the $\mathrm{H} 1$ medium. Following the appropriate incubation period, the microbial cells were harvested by centrifugation, stained with methylene blue and imaged using the Canon Powershot A3300/S Digital Camera (Japan). The microscopes used in this study were the OPTON universal microscope (West Germany) and XSM-20 biological microscope (Ningbo Sunny Instruments Co., Ltd.).

\section{Analytical procedures}

The hydrogen content was determined using a GC-9A gas chromatograph with a thermal conductivity detector (Shimadzu, Japan). The carrier gas was $\mathrm{N}_{2}$, and the injection volume was $1 \mathrm{~mL}$. The hydrogen content was calculated using the external-standard method. Electrochemical parameters were measured using an FJA-3 electrochemical ion analyser (Nanjing Chuan-Di Instrument \& Equipment Co., Ltd.). The fermentation broth was collected periodically, and the $\mathrm{pH} / \mathrm{Eh}$ and electrical conductance/conductivity were determined. The measurements were repeated 5 times, and the average values were used for data analysis.

\section{Competing interests}

The authors declare that they have no competing interests.

\section{Authors' contributions}

PC and HYL initiated and supervised the study. PC,YXW, LY and YQW carried out the the effects of various factors on biohydrogen production studies. SYL, XJY, NBW and NL participated in the small-scale experiments. PC, YXW and $L Y$ draft the manuscript. $P C, Y X W, L Y, Y Q W$ and $H Y L$ revised the manuscript. All authors read and approved the final manuscript.

\section{Acknowledgements}

This work was supported by Gansu Province Science Foundation for Distinguished Young Scholars (Grant No. 1308RJDA014), Longyuan Support Project for Young Creative Talents (Grant No. GANZUTONGZI [2014] no.4), Technology Program of Gansu Province (Grant No. 1205TCYA034), Technology Program of Lanzhou City (Grant No. 2013-4-115), and the National Natural Science Foundation of China (Grant No. 81200469).

\section{Author details}

'School of Pharmacy, Lanzhou University, Donggang West Road No. 199, Lanzhou 730020, People's Republic of China. ${ }^{2}$ Gansu Institute of Business and Technology, Yannan Road No. 449, Lanzhou 730010, People's Republic of China. ${ }^{3}$ Key Laboratory of Fermentation Resources and Application of Institutes of Higher Learning in Sichuan, School of Life Science and Food Engineering, Institute for Bioengeering, Yibin University, Jiusheng Road No. 8, Yibin 644000, People's Republic of China. ${ }^{4}$ College of Life Science and Technology, Heilongjiang Bayi Agricultural University, Daqing 163319, People's Republic of China. ${ }^{5}$ The Reproductive Medicine Research Center of the First Hospital of Lanzhou University, Donggang West Road No. 1, Lanzhou 730020, People's Republic of China. 
Received: 26 June 2014 Accepted: 28 April 2015

Published online: 06 May 2015

\section{References}

1. Patterson T, Esteves S, Dinsdale R, Guwy A, Maddy J. Life cycle assessment of biohydrogen and biomethane production and utilisation as a vehicle fuel. Bioresour Technol. 2013;131:235-45.

2. Pawar SS, van Niel EW. Thermophilic biohydrogen production: how far are we? Appl Microbiol Biotechnol. 2013;97(18):7999-8009.

3. Beckers L, Hiligsmann S, Lambert SD, Heinrichs B, Thonart P. Improving effect of metal and oxide nanoparticles encapsulated in porous silica on fermentative biohydrogen production by Clostridium butyricum. Bioresour Technol. 2013;133:109-17.

4. Boni MR, Sbaffoni S, Tuccinardi L, Viotti P. Development and calibration of a model for biohydrogen production from organic waste. Waste Manag. 2013;33(5):1128-35.

5. Xiao $Y$, Zhang $X$, Zhu M, Tan W. Effect of the culture media optimization, $\mathrm{pH}$ and temperature on the biohydrogen production and the hydrogenase activities by Klebsiella pneumoniae ECU-15. Bioresour Technol. 2013;137:9-17.

6. Zhang X, Ye X, Finneran KT, Zilles JL, Morgenroth E. Interactions between Clostridium beijerinckii and Geobacter metallireducens in co-culture fermentation with anthrahydroquinone-2, 6-disulfonate $\left(\mathrm{AH}_{2} \mathrm{QDS}\right)$ for enhanced biohydrogen production from xylose. Biotechnol Bioeng. 2013;110(1):164-72.

7. Sarkar O, Kannaiah Goud R, Venkata Subhash G, Venkata Mohan S. Relative effect of different inorganic acids on selective enrichment of acidogenic biocatalyst for fermentative biohydrogen production from wastewater. Bioresour Technol. 2013;147:321-31.

8. Yasin NH, Mumtaz T, Hassan MA, Abd Rahman N. Food waste and food processing waste for biohydrogen production: A review. J Environ Manage. 2013;130C:375-85.

9. Mohanraj S, Anbalagan K, Kodhaiyolii S, Pugalenthi V. Comparative evaluation of fermentative hydrogen production using Enterobacter cloacae and mixed culture: Effect of Pd (II) ion and phytogenic palladium nanoparticles. J Biotechnol. 2014;192:87-95.

10. Patel SKS, Kumar P, Singh M, Lee J-K, Kalia VC. Integrative approach to produce hydrogen and polyhydroxybutyrate from biowaste using defined bacterial cultures. Bioresour Technol. 2015;176:136-41.

11. Robledo-Narvaez PN, Munoz-Paez KM, Poggi-Varaldo HM, Rios-Leal E, Calva-Calva G, Ortega-Clemente LA, et al. The influence of total solids content and initial $\mathrm{pH}$ on batch biohydrogen production by solid substrate fermentation of agroindustrial wastes. J Environ Manage. 2013;128:126-37.

12. Guo WQ, Ren NQ, Wang XJ, Xiang WS, Ding J, You Y, et al. Optimization of culture conditions for hydrogen production by Ethanoligenens harbinense B49 using response surface methodology. Bioresour Technol. 2009;100(3):1192-6.

13. Gong ML, Ren NQ, Xing DF. Start-up of bio-hydrogen production reactor seeded with sewage sludge and its microbial community analysis. Water Sci Technol. 2005;52(1-2):115-21.

14. Liu XM, Ren NQ, Song FJ. Recent advances in biohydrogen production by microbe fermentation. Acta Energiae solaris sinica. 2008;05:544-9.

15. Hwang JH, Choi JA, Abou-Shanab RA, Min B, Song H, Kim Y, et al. Feasibility of hydrogen production from ripened fruits by a combined two-stage (dark/dark) fermentation system. Bioresour Technol. 2011;102(2):1051-8.

16. Kongjan PSOT, Angelidaki I. Performance and microbial community analysis of two-stage process with extreme thermophilic hydrogen and thermophilic methane production from hydrolysate in UASB reactors. Bioresour Technol. 2011;102(5):4028-35.

17. Nielsen P, Sørensen J. Multi-target and medium-independent fungal antagonism by hydrolytic enzymes in Paenibacillus polymyxa and Bacillus pumilus strains from barley rhizosphere. FEMS Microbiol Ecol. 1997;22(3):183-92.

\section{Submit your next manuscript to BioMed Central and take full advantage of:}

- Convenient online submission

- Thorough peer review

- No space constraints or color figure charges

- Immediate publication on acceptance

- Inclusion in PubMed, CAS, Scopus and Google Scholar

- Research which is freely available for redistribution

Submit your manuscript at www.biomedcentral.com/submit 